\title{
Closing the Brief Case: The Unexpected Souvenir
}

\author{
aDepartment of Medicine, Division of Infectious Diseases, Mayo Clinic, Phoenix, Arizona, USA \\ bDepartment of Laboratory Medicine and Pathology, Mayo Clinic, Rochester, Minnesota, USA \\ cDepartment of Internal Medicine, Mayo Clinic, Phoenix, Arizona, USA \\ dDepartment of Medicine, Division of Hematology and Oncology, Mayo Clinic, Phoenix, Arizona, USA \\ eDepartment of Laboratory Medicine and Pathology, Mayo Clinic, Phoenix, Arizona, USA
}

Kara Asbury, ${ }^{a}$ Maria Teresa Seville, ${ }^{a}$ Bobbi Pritt, ${ }^{b}$ Allison Scotch, ${ }^{c}$ Allison Rosenthal, d Thomas E. Grys, ${ }^{e}$ Katalin Kelemene

KEYWORDS visceral leishmaniasis, Leishmania infantum, hemophagocytic lymphohistiocytosis, Italy, dogs, leishmaniasis

\section{SELF-ASSESSMENT QUESTIONS}

1. Which is the most sensitive specimen for diagnosing visceral leishmaniasis?
A. Skin biopsy specimen
B. Bone marrow biopsy specimen
C. Liver biopsy specimen
D. Splenic aspiration

Answer: D. Splenic aspiration is the most sensitive specimen to yield the diagnosis of visceral leishmaniasis, with a sensitivity approaching 93\% to 99\% (1). However, it is technically difficult, and there is a risk of significant hemorrhage with capsular tear. Bone marrow aspiration is a safer method to make the diagnosis; however, sensitivity is $52 \%$ to $85 \%$, a reflection of the variable parasitic burden in the bone marrow (1).

2. What is the main differentiating morphological feature of Leishmania species amastigotes that is used to make the diagnosis?
A. Intracellular location
B. Negative GMS stain
C. Rod-shaped kinetoplast
D. Extracellular location

Answer: C. Each amastigote contains a small nucleus with the rod-shaped kinetoplast, which allows differentiation of amastigotes from other small intracellular objects, such as yeasts and T. gondii tachyzoites. The rod-shaped kinetoplast is a DNA-rich structure that, if seen, confirms the diagnosis of leishmaniasis or Chagas disease. Further clinical, histologic, and epidemiologic features can then be used to differentiate these two diseases. See Table 3 in the Brief Case.

3. What is the first-line treatment of symptomatic visceral leishmaniasis in North America?
A. Pentamidine
B. Miltefosine
C. Pentavalent antimony
D. Liposomal amphotericin B

Answer: D. Per IDSA guidelines, liposomal amphotericin B is the treatment of choice for visceral leishmaniasis in both immunocompetent and immunocom-

Citation Asbury K, Seville MT, Pritt B, Scotch A Rosenthal A, Grys TE, Kelemen K. 2018. Closing the Brief Case: The unexpected souvenir. J Clin Microbiol 56:e01414-17. https://doi.org/10 .1128/JCM.01414-17.

Editor Carey-Ann D. Burnham, Washington University School of Medicine Copyright $\odot 2018$ American Society for Microbiology. All Rights Reserved.

Address correspondence to Kara Asbury, Asbury.Kara@mayo.edu.

See https://doi.org/10.1128/JCM.01387-17 in this issue for case presentation and discussion. 
promised patients in North America. Miltefosine and pentavalent antimony are still used in the treatment of visceral leishmaniasis in certain locations, including Africa, South America, and Bangladesh, based on drug availability and resistance patterns. Miltefosine resistance has been seen in India and Nepal; in North Bihar, there are pentavalent antimony drug failures. Both miltefosine and pentavalent antimony are contraindicated in pregnant individuals.

\section{TAKE-HOME POINTS}

- Leishmaniasis is one of the rare arthropod-borne parasitic causes of secondary hemophagocytic lymphohistiocytosis.

- There are three primary clinical syndromes of leishmaniasis: visceral, cutaneous, and mucosal diseases. The causative Leishmania spp., anthroponotic vector, and mammalian reservoirs vary by geographic location.

- Visceral leishmaniasis is a result of dissemination of parasites of the Leishmania donovani complex, typically Leishmania donovani or Leishmania infantum/Leishmania chagasi, within the reticuloendothelial system. Phlebotomine sandflies, Lutzomyia (in the Americas) and Phlebotomus (everywhere else), transmit the parasite. Humans, rodents, and canines can serve as reservoirs.

- Leishmania is endemic in the Mediterranean basin, with dogs serving as the main reservoir host.

- The diagnosis relies upon recognition of amastigotes containing a small nucleus and a rod-shaped kinetoplast, found only within macrophages. This is a main differentiating feature from $T$. cruzi, whose amastigotes can be found in any nucleated cell.

- Liposomal amphotericin B is the treatment of choice for visceral leishmaniasis in North America.

\section{REFERENCE}

1. Aronson N, Herwaldt B, Libman M, Pearson R, Lopez-Velez R, Weina $P$, Carvalho E, Ephros M, Jeronimo S, Magill A. 2016. Diagnosis and treatment of leishmaniasis: clinical practice guidelines by the Infectious Dis- eases Society of America (IDSA) and the American Society of Tropical Medicine and Hygiene (ASTMH). Clin Infect Dis 63:e202-e264. https://doi .org/10.1093/cid/ciw670. 